\title{
Central Centrifugal Cicatricial Alopecia Presenting with Irregular Patchy Alopecia on the Lateral and Posterior Scalp
}

\author{
Mariya Miteva Antonella Tosti \\ Department of Dermatology and Cutaneous Surgery, University of Miami Miller School of Medicine, Miami, Fla., USA
}

\section{Key Words}

Hair loss · African-American · Black scalp · Dermatoscopy ·

Trichoscopy $\cdot$ Pathology $\cdot$ Scarring

\begin{abstract}
Background: Central centrifugal cicatricial alopecia (CCCA) is the most common cause of scarring alopecia among women of African descent which affects the central scalp and spreads centrifugally but spares the lateral and posterior scalp. Objectives: The objective of this study is to report on a new clinical variety of CCCA presenting with patchy alopecia involving the lateral and posterior scalp in addition to the central scalp. Materials and Methods: We reviewed the medical records and the clinical, dermatoscopic and pathologic data of 14 African-American women with CCCA presenting with patchy alopecia. Results: Two patients had individual well-delineated patches of hair loss, and 12 patients had multiple irregular patches of hair loss. In all cases, the alopecia affected the lateral and posterior scalp in addition to the central scalp. Four patients had a history of traumatic hairstyles, and 10 patients were also affected by marginal traction alopecia. On dermatoscopy, the patches showed peripilar white-gray halos and broken hairs. The pathologic diagnosis of CCCA was based on the following features: fol-
\end{abstract}

licular dropout, absent or only focally preserved sebaceous glands, premature desquamation of the inner root sheath and perifollicular fibrosis with mild inflammatory infiltrate. Conclusion: CCCA can present with patches of hair loss involving the parietal and posterior scalp in addition to the central scalp. Without dermatoscopy and pathology, this variety can be easily misdiagnosed as traction alopecia.

(c) 2015 S. Karger AG, Basel

\section{Introduction}

Central centrifugal cicatricial alopecia (CCCA) is the most common cause of scarring alopecia among women of African descent [1]. It was first described by Lo Presti et al. [2] in 1968 as 'hot comb alopecia' or irreversible alopecia of the scalp in black women using hot petrolatum while hot combing. It was later named 'follicular degeneration syndrome' to reflect the common pathologic finding in the affected follicles: loss of the inner root sheath and migration and destruction of the hair shafts in the dermis [3]. Over the decades, more than 50 studies have been published, all of which describe the same clinical presentation: chronic and progressive central scalp hair loss which starts on the crown and spreads peripher-

\section{KARGER 125}

(c) 2015 S. Karger AG, Base

2296-9195/15/0011-0001\$39.50/0

E-Mail karger@karger.com

www.karger.com/sad
Mariya Miteva, MD

University of Miami Miller School of Medicine

1600 NW 10th Avenue, RSMB, Room 2023A

Miami, FL 33136 (USA)

E-Mailmmiteva@med.miami.edu 
Fig. 1. a, b Photograph of an AfricanAmerican woman with CCCA. a Multiple patches of alopecia with cribriform arrangement involving the lateral and posterior scalp are shown. b Dermatoscopy shows pinpoint white dots, irregular white patches, peripilar white-gray halos and a black dot (Handyscope; FotoFinder Systems, Bad Birnbach, Germany; HE; $\times 20$ ).
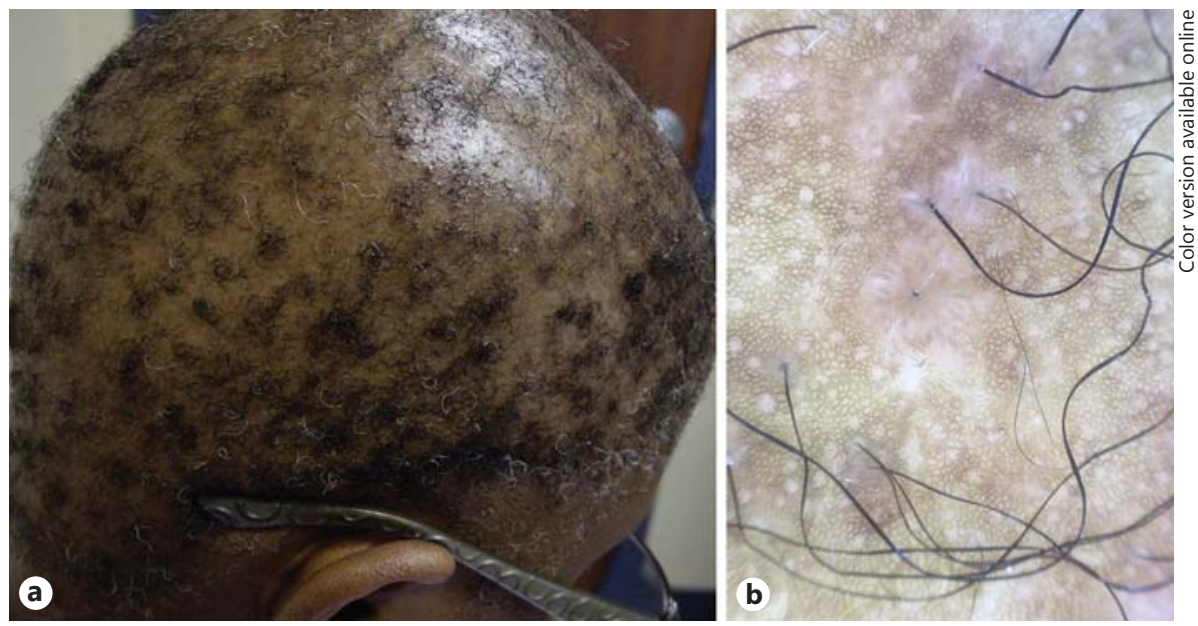

ally but spares the lateral and posterior scalp [4]. Advanced cases show a smooth and shiny scalp. Recently, hair breakage on the vertex has been reported as a possible early clinical presentation that was confirmed on pathology [5]. The North American Hair Research Society (NAHRS) created the name CCCA in 2011 to specifically reflect the characteristic central pattern of spreading hair loss. A central scalp alopecia photographic scale ranging from 0 to 5 is utilized to accentuate the more severely affected anatomical area: the frontal scalp (A subtype) or the vertex (B subtype) [1].

We report on a new clinical variety of CCCA presenting with patches of hair loss affecting the lateral and posterior scalp.

\section{Materials and Methods}

Medical records and clinical and dermatoscopic images of 14 patients with patchy CCCA were reviewed in order to collect demographic data, hairstyle preferences and clinical features. All patients had been seen as outpatients at the University of Miami in the previous 2 years. In all of them, the diagnosis of CCCA was confirmed by a scalp biopsy obtained from the lateral and/or posterior scalp. The pathologic specimens were reassessed for diagnostic features on horizontal sections stained with hematoxylin and eosin. This study was approved by the institutional review board.

\section{Case Report}

Patients were African-American women with a mean age ranging from 18 to 59 years. The duration of disease varied between 8 months and 5 years. All patients had a documented history of hair grooming and traumatic hairstyles: braids and/or cornrows from a young age $(\mathrm{n}=14)$ and hair weaving (addition of exogenous hu-

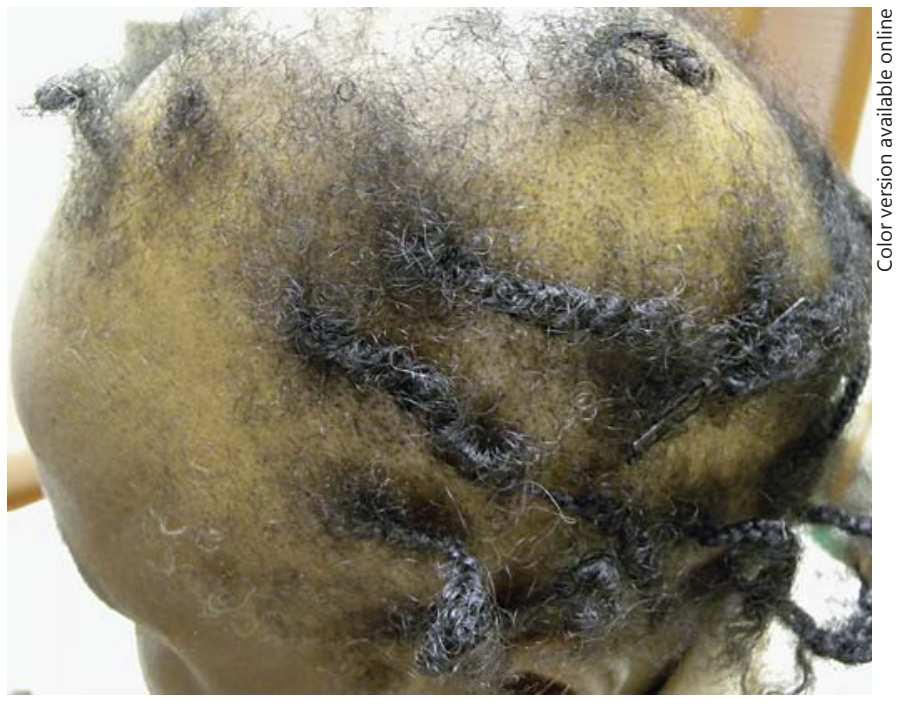

Fig. 2. Photograph of another case of an African-American woman with patchy hair loss among braided hair.

man or synthetic hair by sewing or bonding with glue) at some points of their life $(n=3)$. All patients had a long history of chemical straightening with relaxers but reported no ongoing use. At the time of presentation, 4 of them had a braided or cornrowed hairstyle and 10 had natural hair; 8 of them were wearing a wig. Only 3 reported occasional scalp itching and pain. Eleven had no previous biopsy or treatment, and 3 had been treated elsewhere with topical minoxidil and intralesional steroid injections for traction alopecia with possible CCCA.

Clinically, all 14 patients presented with patches of hair loss on the parietal (lateral) and posterior scalp in addition to the central involvement. There were two clinical presentations: (1) 2 patients 
had individual well-delineated patches of hair loss on the background of overall preserved hair density, and (2) 12 patients had numerous interconnected irregular patches of hair loss resembling the irregular maze-like growth of moss on a stone (fig. 1a, 2). In patients with braided hairs, the patches were difficult to appreciate prior to the removal of the braids (fig. 3).

Marginal traction alopecia was present in 10 of the 14 cases. Dermatoscopy of the affected areas showed a scarring alopecia pattern with pinpoint white dots and irregular white patches. Presence of white-gray halos highly suggested a possible diagnosis of CCCA. Other features included hair shaft variability, broken hairs (6 patients), scaling and hair casts ( 2 patients) (fig. $1 \mathrm{~b})$.

Skin, mouth and nail examinations were negative in all patients. All specimens revealed diagnostic features of CCCA: (1) reduced follicular density; (2) altered follicular architecture with areas of follicular dropout and absent or only focally preserved sebaceous glands; (3) premature desquamation of the inner root sheath; (4) individual or compound follicular structures surrounded by perifollicular fibrosis and mild inflammatory infiltrate, and (5) naked hair shafts in the dermis (fig. 4).

\section{Discussion}

We describe a new clinical presentation of CCCA in which individual or multiple alopecic patches with a cribriform arrangement involve the parietal and posterior scalp in addition to the typical central location. All of our 14 cases showed dermatoscopic and histopathologic findings supporting the diagnosis of CCCA $[6,7]$.

The main differential diagnosis of patchy CCCA includes other patchy hair disorders such as traction alopecia, which in our series was commonly associated, alopecia areata, lichen planopilaris (LPP), discoid lupus erythematosus (DLE) and tinea capitis. Dermatoscopy and pathology are necessary to make the correct diagnosis. Table 1 summarizes the main distinguishing clinical, dermatoscopic and pathologic features. Clinically, the main difference between patchy CCCA and LPP is the presence of vellus hairs within the patches, which are absent in LPP and frontal fibrosing alopecia. Dermatoscopic features that characterize LPP and/or DLE, including peripilar casts, follicular plugging and red dots, are not found [8]. Patchy CCCA is clinically similar to traction alopecia. However, dermatoscopy helps distinguishing the diseases, as it shows peripilar whitegray halos only in CCCA. Alopecia areata and tinea capitis are easily excluded on dermatoscopy by the presence of different types of dystrophic hairs in alopecia areata and comma and corkscrew hairs in tinea capitis. On pathology, the association of premature desquamation of the inner root sheath, focal preservation of sebaceous glands and the presence of compound follicular

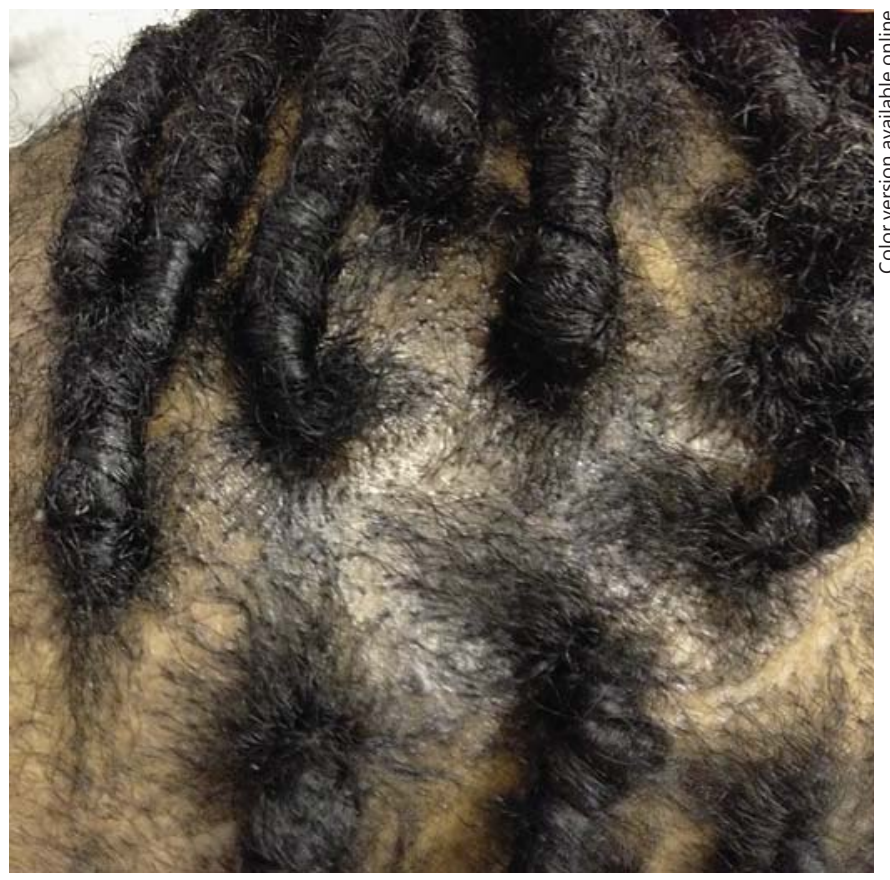

Fig. 3. Photograph of a patient with CCCA presenting initially with tight braided hair. This pattern can be clinically misdiagnosed as traction alopecia if dermatoscopy and pathology are not utilized.

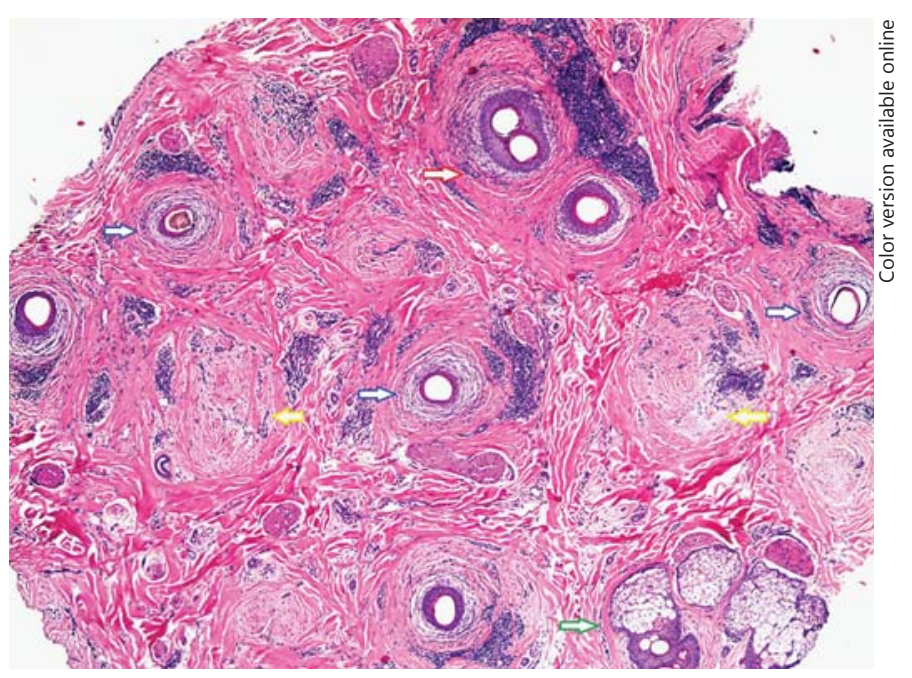

Fig. 4. A horizontal section of a scalp biopsy obtained from the patient of figure 1 is presented. Overall decreased follicular density (a total of 8 follicles) and altered follicular architecture with areas of follicular scarring (yellow arrows) are observed. The remaining follicles show no inner root sheath and are surrounded by perifollicular fibrosis and moderate lichenoid inflammation (blue arrows). A compound follicular structure showing the same involvement is present (red arrow). The sebaceous glands are only focally preserved (green arrow). HE; $\times 4$. Colors refer to the online version only. 
Table 1. Summary of the distinguishing clinical, dermatoscopic and pathologic findings in patchy hair disorders among women of African descent

\begin{tabular}{|c|c|c|}
\hline Patchy hair disorder & Scalp dermatoscopy & Pathology \\
\hline CCCA & Peripilar white-gray halo & $\begin{array}{l}\text { Follicular dropout } \\
\text { Compound follicular structures with perifollicular fibrosis } \\
\text { and usually mild inflammatory infiltrate } \\
\text { Premature desquamation of the inner root sheath } \\
\text { Focal preservation of sebaceous glands } \\
\text { Naked hair shafts }\end{array}$ \\
\hline Alopecia areata & $\begin{array}{l}\text { Exclamation mark hairs } \\
\text { Dystrophic hairs }\end{array}$ & $\begin{array}{l}\text { Nonscarring alopecia } \\
\text { 'Swarm of bees' peribulbar infiltrate }\end{array}$ \\
\hline Traction alopecia & Nondiagnostic & $\begin{array}{l}\text { Intact sebaceous glands } \\
\text { No perifollicular fibrosis } \\
\text { No perifollicular inflammation }\end{array}$ \\
\hline LPP & Peripilar casts & $\begin{array}{l}\text { Follicular dropout } \\
\text { Perifollicular lichenoid infiltrate with fibrosis } \\
\text { Absence of sebaceous glands }\end{array}$ \\
\hline DLE & $\begin{array}{l}\text { Peripilar casts } \\
\text { Follicular plugs } \\
\text { Red dots }\end{array}$ & $\begin{array}{l}\text { Follicular dropout } \\
\text { Perifollicular/periadnexal interface inflammatory infiltrate } \\
\text { with fibrosis } \\
\text { Junctional interface involvement }\end{array}$ \\
\hline Tinea capitis & $\begin{array}{l}\text { Comma hairs } \\
\text { Corkscrew hairs }\end{array}$ & $\begin{array}{l}\text { Fungal hyphae and spores within affected hair shafts on } \\
\text { PAS stain }\end{array}$ \\
\hline
\end{tabular}

structures with hyper-/parakeratosis in the hair canal and only mild inflammation favors the diagnosis of CCCA. In particular, LLP can be excluded by the absence of perifollicular lichenoid infiltrate and junctional involvement. Although CCCA biopsies may show inflammatory infiltrate, this is usually sparse and does not affect the dermoepidermal junction [9]. DLE is excluded by the absence of junctional interface involvement and periadnexal infiltrate. Traction alopecia shows preservation of the sebaceous glands and no signs of follicular destruction such as perifollicular fibrosis and inflammation.

All our patients had a history of (or were still wearing) heavy traction-inducing hair styles such as glued weaves, dreadlocks and braids. Traction alopecia due to traumatic hairstyles is very common among women of African descent [10]. It usually presents with marginal alopecia involving the frontoparietal scalp but also may show as patches of alopecia on the rest of the scalp. In our experience, traction alopecia is common among women with CCCA, including patchy CCCA, although a large study from South Africa did not find a single woman with
CCCA among over 1,000 participants with traction alopecia [11]. A possible explanation is that this study did not involve pathologic confirmation.

In conclusion, we reported on a new clinical presentation of CCCA with patches of alopecia which can involve the parietal and posterior scalp. The severity of this variety cannot be classified using the available central scalp alopecia photographic scale [1]. In women of African descent who complain of hair loss but are reluctant to remove the braids or weaves, the scalp cannot be properly evaluated for CCCA, and their disease may be misdiagnosed as traction alopecia.

\section{Disclosure Statement}

The authors have no conflicts of interest to disclose.
Miteva/Tosti 


\section{References}

1 Olsen EA, Callender V, Sperling L, et al: Central scalp alopecia photographic scale in African American women. Dermatol Ther 2008; 21:264-267.

$>2$ LoPresti P, Papa CM, Kligman AM: Hot comb alopecia. Arch Dermatol 1968;98:234-238.

$>3$ Sperling LC, Sau P: The follicular degeneration syndrome in black patients. 'Hot comb alopecia' revisited and revised. Arch Dermatol 1992;128:68-74.

4 Olsen EA, Callender V, McMichael A, et al: Central hair loss in African American women: incidence and potential risk factors. J Am Acad Dermatol 2011;64:245-252.
5 Callender VD, Wright DR, Davis EC, Sperling LC: Hair breakage as a presenting sign of early or occult central centrifugal cicatricial alopecia: clinicopathologic findings in 9 patients. Arch Dermatol 2012;148:1047-1052.

6 Miteva M, Tosti A: Dermatoscopic features of central centrifugal cicatricial alopecia. J Am Acad Dermatol 2014;71:443-449.

7 Miteva M, Tosti A: 'A detective look' at hair biopsies from African-American patients. $\mathrm{Br}$ J Dermatol 2012;166:1289-1294.

8 Miteva M, Tosti A: Hair and scalp dermatoscopy. J Am Acad Dermatol 2012;67:10401048 .
9 Miteva M, Tosti A: Pathologic diagnosis of central centrifugal cicatricial alopecia on horizontal sections. Am J Dermatopathol 2014; 36:859-867.

10 Khumalo NP, Jessop S, Gumedze F, Ehrlich R: Determinants of marginal traction alopecia in African girls and women. J Am Acad Dermatol 2008;59:432-438.

11 Khumalo NP, Gumedze F: Traction: risk factor or coincidence in central centrifugal cicatricial alopecia? Br J Dermatol 2012;167: 1191-1193. 